\title{
Optimal spatial-dynamic management to minimize the damages caused by aquatic invasive species
}

\author{
Katherine Y. Zipp · Yangqingxiang Wu · Kaiyi Wu · Ludmil \\ T. Zikatanov
}

Received: date / Accepted: date

\begin{abstract}
Invasive species have been recognized as a leading threat to biodiversity. In particular, lakes are especially affected by species invasions because they are closed systems sensitive to disruption. Accurately controlling the spread of invasive species requires solving a complex spatial-dynamic optimization problem. In this work we propose a novel framework for determining the optimal management strategy to maximize the value of a lake system net of damages from invasive species, including an endogenous diffusion mechanism for the spread of invasive species through boaters' trips between lakes. The proposed method includes a combined global iterative process which determines the optimal number of trips to each lake in each season and the spatial-dynamic optimal boat ramp fee.
\end{abstract}

Keywords Invasive species · Spatial-dynamic management

JEL Subject Classification Q20 · Q50 - Q57

\section{Introduction}

Globally, invasive species have long been recognized as a leading threat to biodiversity [1,2]. Lakes are especially affected by species invasions because they are closed systems sensitive to disruption [2,3]. As a result, controlling the spread of aquatic invasive species (AIS) has been a major management effort for the past two decades. Further complicating AIS management, AIS are largely spread inadvertently through the movement of recreational boaters from lake to lake [4]. Therefore, management is tasked with maximizing the value of a lake system net of AIS damages by changing boating behavior and thus the spread of AIS.

The work of Zipp and Y. Wu was partially supported by the Department of Agricultural Economics, Sociology, and Education at Penn State. The work of Zikatanov was partially supported by NSF grants DMS-1720114 and DMS-1819157.

Katherine Y. Zipp

Department of Agricultural Economics, Sociology and Education, Penn State, University Park, PA 16802

E-mail: kyz1@psu.edu

Yangqingxiang $\mathrm{Wu}$

Department of Mathematics, Penn State, University Park, PA 16802

E-mail: yzw137@psu.edu

Kaiyi Wu

Department of Mathematics, Tufts University, Medford, MA 02155

E-mail: Kaiyi.Wu@tufts.edu

Ludmil T. Zikatanov

Department of Mathematics, Penn State, University Park, PA 16802

E-mail: ludmil@psu.edu 
In this article, we develop a novel spatial-dynamic framework to determine the optimal policy to induce the optimal number of trips to each lake in a system across many seasons to maximize the net benefits of the lake system taking into consideration the damages from the spread of invasive species. This policy must be heterogeneous across space and time. Spatially, it must depend on boating patterns (which depend on the attractiveness of lakes, substitutability across lakes, the presence of invasive species) and the ecological suitability of a lake for invasion. Temporally, it must take into account the time dependent distribution of AIS across the system. AIS spread is a vicious cycle, as an increase in invaded lakes provides more opportunity for spread to uninvaded lakes. The pathways for dispersal of AIS depend on boating behavior, which in turn depends on lake management and the presence or absence of AIS in each lake. This leads to a complicated feedback loop where the optimal policy depends on the dispersal of AIS and the number of trips to each lake, the dispersal of AIS depends on boating behavior, and boating behavior depends on policy and the dispersal of AIS (see Figure 1). The dispersal of AIS and boating behavior both depend on the presence or absence of AIS in every lake in the system. Therefore, the optimal policy must be globally coordinated across the system [5].

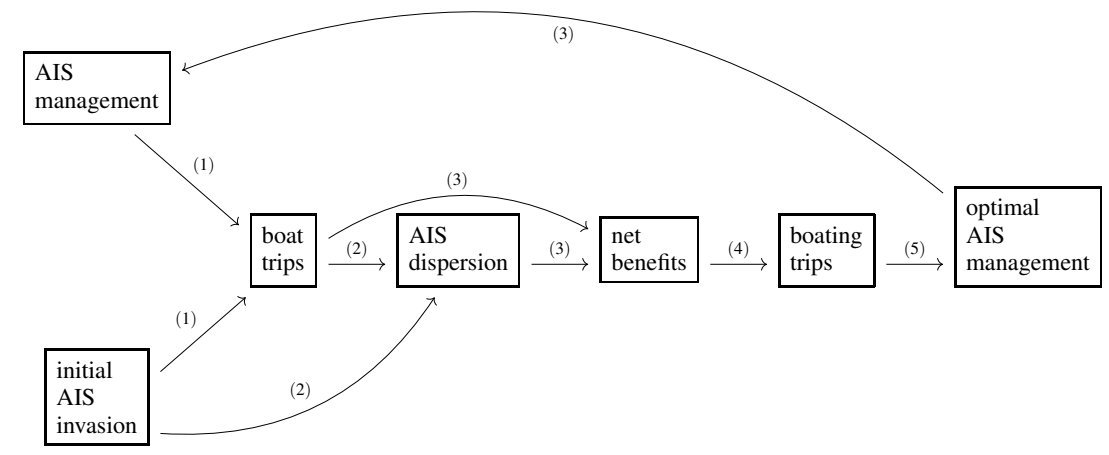

Fig. 1 Diagram of model framework

There are five major components to our optimal spatial-dynamic AIS management framework.

1. Model of recreational boating decisions. The dispersal of AIS depends on boating decisions, therefore, we need a model of how boaters choose where to boat. We rely on a standard economic model known as a random utility model (RUM) in which boaters maximize their utility by choosing where to boat.

2. Model of AIS dispersal. The dispersal of AIS requires (i) boaters to visit an invaded lake, (ii) inadvertently transport the invasive species out of the invaded lake, (iii) then visit an uninvaded lake while the invaded species is still alive, and finally (iv) the invasive species must become established in a suitable lake [6]. Given the dispersal of AIS, we model the probability that a lake becomes invaded given that it is not already invaded as a hazard model.

3. Net benefits to recreational boaters. Lakes provide benefits to boaters. Our RUM allows us to calculate the welfare benefits of boating. The net benefits of the lake system are these benefits of boating minus the damages caused by AIS. We consider both the damages from AIS to both shoreline property owners [7, 8] and boaters [9].

4. Optimal number of boating trips to each lake. We find the optimal number of boating trips to each lake in each season to maximize net benefits (step 3) subject to AIS dispersal (step 2) and the model of boating decisions (step 1).

5. Optimal policy. Finally, we find the optimal policy that leads boaters to choose the socially optimal number of trips.

This framework makes three major contributions to the literature. First, we develop a method that has not been used in the emerging literature on optimal spatial-dynamic policy management. 
[5, 10, 11, 12, 13, 14, 15, 16]. Previous literature has focused on dynamic optimization procedures that are plagued by the curse of dimensionality. Our framework does not suffer this problem and does not require large computing power to implement. Second, we incorporate an endogenous dispersal method. The literature on optimal spatial-dynamic bioeconomic policies typically models the dispersal of species as radially dispersing from an original location. Our model on AIS allows the dispersal mechanism to depend on the movement of boaters and thus be endogenous. Third, we have a fully-coupled natural human system where boating decisions depend on the status of invasions and the dispersal of invasions depends on boating decisions.

\section{Problem Formation}

The spatial-dynamic optimal policy can be determined through an iterative process with five major components (see Figure 1), which will be explained in detail.

\subsection{Model of recreational boating decisions}

We model boating decisions as a repeated random utility travel cost model in which boaters $n \in\{1, \ldots, N\}$ maximize their utility $U_{\text {isnt }}$ on day $t \in\{1, \ldots, T\}$ in season $s \in\{1, \ldots, S\}$ by either (a) visiting lake $i \in$ $\{2, \ldots, I\}$; or (b) choosing not to go boating (denoted by $i=1$ ). Let utility be defined as

$$
\begin{aligned}
& U_{i s n t}=v_{i s n t}+\varepsilon_{i s n t}, \quad \text { where } \\
& v_{i s n t}=Z_{i s n t}+\alpha\left(M_{n}-\tau_{i, s}\right)-\xi x_{i(s-1)},
\end{aligned}
$$

$Z_{i s n t}=\sum_{m} \beta_{m} z_{i s n t, m}$ represents $m$ boater, lake, day, or season attributes $\left(z_{i s n t, m}\right)$ that influence decision to go boating or stay home with parameters $\beta_{m}$ and remains constant in our model, $\alpha$ is marginal utility of income, $M_{n}$ is the income of boater $n, \tau_{i s}$ is the boat ramp fee, $\xi$ is the effect of AIS on boater utility, $x_{i(s-1)}$ is the status of AIS lake $i$ at the end of the previous season $(s-1)$, and $\varepsilon_{i s n t}$ are identically independently distributed Type I Extreme Value random variables with variance $\pi^{2} / 6$. Under this assumption of error distribution, the probability that boater $n$ chooses an alternative $i \in\{1, \ldots I\}$ on day $t$ in season $s$ is given by the conditional logit model [17]

$$
P_{i s n t}=\frac{\exp \left(v_{i s n t}\right)}{\sum_{j=1}^{I} \exp \left(v_{j s n t}\right)} .
$$

We set the total number of trips taken to lake $i \in\{2, \ldots, I\}$ (or days spent at home in the case of $i=1$ ) in season $s$ to be $b_{i s}=\sum_{n=1}^{N} \sum_{t=1}^{T} P_{i s n t}$ (note that $\sum_{i=1}^{I} b_{i s}=N T$ by definition). Clearly, $b_{i s}$ is a function of the boat ramp fees $\left\{\tau_{j s}\right\}_{j=2}^{I}$ and the invasion statuses $\left\{x_{j(s-1)}\right\}_{j=2}^{I}$.

\subsection{Model of AIS dispersal}

AIS are largely spread inadvertently through the movement of boaters across lakes [18,4]. We define the status of the invasion in lake $i$ in season $s$, denoted by $x_{i s}$, as the probability that lake $i$ is invaded at the end of season $s$. We assume that invasions are irreversible so that once a lake becomes invaded it remains invaded for all remaining seasons. Let $\widetilde{x}_{i s}$ be the probability that lake $i$ is invaded at the end of season $s$ conditional on being uninvaded at the end of season $s-1$. We have the following relations.

$$
x_{i(s+1)}=1-\prod_{m=1}^{s}\left(1-\tilde{x}_{i m}\right), \quad \text { or equivalently, } \quad x_{i, s+1}=x_{i s}+\left(1-x_{i s}\right) \tilde{x}_{i(s+1)}
$$

\footnotetext{
${ }^{1}$ We assume that the invasion status is updated at the end of the season such that boaters' trip decisions depend on $x_{s-1}$ and at the end of the season when the invasion status is updated $x_{s}$ depends on the boating decisions in season $s$.
} 
We assume that $x_{i, s=1}=: x_{i 1}$ is given. We use the following approximate hazard model:

$$
\widetilde{x}_{i s}=\mathfrak{s}\left(K_{i s}\right)= \begin{cases}0, & K_{i s}<-a, \\ \frac{K_{i s}+a}{2 a}, & K_{i s} \in[-a, a], \\ 1, & K_{i s}>a,\end{cases}
$$

which approximates the sigmoid function ${ }^{2}$. Here, $K_{i s}$ is defined as

$$
K_{i s}=\gamma L_{i s}+\lambda_{i s}
$$

where $L_{i s}$ is the expected number of trips carrying AIS to lake $i$ in season $s$ (referred to as propagule pressure), $\lambda_{i s}$ is a measure of the suitability of lake $i$ to host AIS, and $\left(\gamma, \xi_{l}\right)$ are estimated parameters related to the effects of propagule pressure and lake suitability on the probability of invasion. Propagule pressure requires (i) boaters to visit an invaded lake, (ii) inadvertently transport the invasive species out of the invaded lake, and (iii) then visit an uninvaded lake while the invaded species is still alive. Recent evidence suggests that the majority of AIS remains alive for approximately one day [19]. It is not possible to spread the invasive species from lake $i$ to itself, so we are only concerned with trips between different lakes. Therefore, the expected probability that a boater visits any invaded lake $j \neq i$ on the previous day is $\sum_{j \neq i} P_{j s n(t-1)} x_{j(s-1)}$. Let $\mu$ be the probability of a boat leaving an invaded lake with AIS (this is a constant independent of $n, i, s, t$ ). Therefore, the propagule pressure is

$$
L_{i s}=\sum_{t=2}^{T} \sum_{n=1}^{N} \underbrace{\left(\mu \sum_{j \neq i} P_{j s n(t-1)} x_{j(s-1)}\right)}_{Q_{i s n t}} P_{i s n t}
$$

where $Q_{i s n t}$ is the expected number of trips that boater $n$ makes to lake $i$ on day $t$ in season $s$ carrying an invasive species from any lake $j \neq i$.

\subsection{Net benefits}

Lakes provide benefits to boaters who receive utility from boating instead of staying home. Let $w_{\text {snt }}=$ $\max \left\{v_{1 s n t}, \ldots, v_{\text {Isnt }}\right\}$. Without any boating, boaters just receive utility from staying home $w_{\text {snt }}=v_{1 \text { snt }}$. Therefore, the value of having the option to boat at all lakes in the system $i \in 2, \ldots, I$ is worth $\mathscr{L}_{\text {snt }}^{s}$ per boater per trip per season:

$$
\begin{aligned}
\mathscr{L}_{\text {snt }}^{s} & =-\frac{1}{|\alpha|}\left(\ln \left(\exp \left(v_{1 \text { snt }}\right)\right)-\ln \left(\sum_{i=1}^{I} \exp \left(v_{\text {isnt }}\right)\right)\right) \\
& =-\frac{1}{|\alpha|}\left(\ln \left(\frac{\exp \left(v_{1 \text { snt }}\right)}{\sum_{i=1}^{I} \exp \left(v_{\text {isnt }}\right)}\right)\right) \\
& =-\frac{1}{|\alpha|}\left(\ln \left(P_{1 \text { snt }}\right)\right) \geq 0,
\end{aligned}
$$

where $\alpha$ is the marginal utility of income and is used to convert measures of utility into measures of dollars and $P_{1 \text { snt }} \leq 1$ is the probability of boater $n$ choosing to stay home $i=1$ on day $t$ in season $s$. The total value of the system to all boaters across all trips in season $s$ is the sum of $\mathscr{L}_{s n t}^{s}$ over boaters $n$ and days $t$ :

$$
\mathscr{W}_{s}^{s}=-\frac{1}{|\alpha|} \sum_{n, t} \ln \left(P_{1 s n t}\right) \approx-\frac{1}{|\alpha|} \sum_{n, t}\left(P_{1 s n t}-1\right)=-\frac{1}{|\alpha|}\left(b_{1 s}-N T\right)=\frac{1}{|\alpha|} \sum_{i=2}^{I} b_{i s} .
$$

The net benefits of the lake system are these benefits $\mathscr{W}_{s}^{s}$ minus the damages caused by AIS. Aquatic invasive species (AIS) cause damage to both shoreline property owners [8,7] and recreational users (e.g.

2 We take $a=2.824153$ which gives an approximates the sigmoid with $\ell_{\infty}$ error at most 0.056075 . 
boaters) [9]. We consider $h_{i}$ shoreline properties around lake $i$, assuming that shoreline properties are constant across seasons. We assume that shoreline property owners incur a constant annual welfare loss, $\mathscr{L}^{h}$, per shoreline property, $h_{i}$, for each invaded lake. Therefore, we define the total welfare loss in season $s$ from the spread of AIS to shoreline property owners as

$$
\mathscr{W}_{s}^{h}=\sum_{i=2}^{I} \mathscr{L}^{h} h_{i} x_{i s}
$$

where we use that $x_{1 s}=0$ for all $s$.

Next, we define the welfare loss from the spread of AIS to boaters. We assume that boaters incur a constant welfare loss, $\mathscr{L}^{b}$, per trip $b_{i s}$ to an invaded lake in season $s$. Therefore, we define the expected total welfare loss in season $s$ from the spread of AIS to recreational boaters as

$$
\mathscr{W}_{s}^{b}=\sum_{i=2}^{I} \mathscr{L}^{b} b_{i s} x_{i s} .
$$

Therefore, the net benefits of the entire lake system is the discounted sum of the benefits of the lake system, $\widetilde{\mathscr{W}_{s}^{s}}$ minus the damages from AIS, $\mathscr{W}_{s}^{h}+\mathscr{W}_{s}^{b}$ :

$$
\mathscr{F}=\sum_{s} \rho^{s}\left(\widetilde{\mathscr{W}_{s}^{s}}-\mathscr{W}_{s}^{h}-\mathscr{W}_{s}^{b}\right)
$$

where $\rho^{s}=\left(\frac{1}{1+r}\right)^{s}$ is the discount factor and $r$ is the discount rate.

\subsection{Optimal number of boating trips to each lake}

With this net benefit function, $\mathscr{F}$, our objective is to find the optimal boat ramp fee $\tau_{i s}^{*}$ that maximizes the net benefit of the lake system subject to the dispersal function of AIS (10). This is a difficult optimization problem to solve. We make two contributions that allow us to more easily solve this problem. First, we rewrite the objective function $\mathscr{F}(\boldsymbol{b}(\boldsymbol{x}, \boldsymbol{\tau}), \boldsymbol{x}(\boldsymbol{b}, \boldsymbol{\tau}))$ as a function of $\boldsymbol{b}$ in the following general form

$$
\mathscr{F}(\widetilde{\boldsymbol{b}}, \boldsymbol{b})=\langle\mathbb{D}(\widetilde{\boldsymbol{b}}) \boldsymbol{b}, \boldsymbol{b}\rangle+2\langle\boldsymbol{f}(\widetilde{\boldsymbol{b}}), \boldsymbol{b}\rangle+\langle\boldsymbol{g}(\widetilde{\boldsymbol{b}}, \boldsymbol{P}), \mathbf{1}\rangle .
$$

The reason this is can be written as function of $\boldsymbol{b}$ is because, as discussed after Algorithm 4, the boat ramp fee $\boldsymbol{\tau}$ in the model can be determined by the number of trips $\boldsymbol{b}$, and the probabilities of invasion $\boldsymbol{x}$ are also uniquely determined from the taxes $\tau$. It is important to note that in general we would like to maximize $\mathscr{F}(\cdot, \cdot)$ above when $\widetilde{\boldsymbol{b}}=\boldsymbol{b}$. Thus, for a fixed $\widetilde{\boldsymbol{b}}$ we have the following maximization to find the optimal number of trips to each lake $b^{*}$ to maximize net benefits with three constraints:(i) AIS dispersal follows (10), (ii) the total number of trips has to equal the number of trip occasions (9), (iii) the minimum number of trips boaters can take to lake $i$ is zero (9).

\section{Optimization Problem}

Find $\quad \boldsymbol{\tau}_{*}:=\arg \max _{\boldsymbol{\tau}} \mathscr{F}(\boldsymbol{b}(\boldsymbol{\tau}), \boldsymbol{P}(\boldsymbol{\tau})=\{\langle\mathbb{D}(\boldsymbol{b}) \boldsymbol{b}, \boldsymbol{b}\rangle+2\langle\boldsymbol{f}(\boldsymbol{b}), \boldsymbol{b}\rangle+\langle g(\boldsymbol{b}, \boldsymbol{P}(\boldsymbol{\tau})), \mathbf{1}\rangle\}$,

Subject to the constraints:

$$
\sum_{i=1}^{I} b_{i s}=N \times T, \quad 0 \leq b_{i s}
$$

where $x_{i s}$ is computed with given initial values $\left\{x_{i 1}\right\}_{i=1}^{I}$ and

$$
x_{i s}=x_{i, s-1}+\left(1-x_{i, s-1}\right) \mathfrak{s}\left(K_{i s}\right)
$$

for $i=2, \ldots, I$ and $s=2, \ldots, S$. 
In what follows we will use iterative approximation of $\mathscr{F}$ by quadratic forms which can be easily optimized. In such cases we evaluate all terms such as $\mathbb{D}(\boldsymbol{b}), \boldsymbol{f}(\boldsymbol{b}), \boldsymbol{g}(\boldsymbol{b}, \boldsymbol{P})$ at our previous iterates, denoted here by $\widetilde{\boldsymbol{b}}, \tilde{\boldsymbol{\tau}}$. We thus have

$$
\mathscr{F}(\widetilde{\boldsymbol{b}}, \boldsymbol{b}):=\langle\mathbb{D}(\widetilde{\boldsymbol{b}}) \boldsymbol{b}, \boldsymbol{b}\rangle+2\langle\boldsymbol{f}(\widetilde{\boldsymbol{b}}), \boldsymbol{b}\rangle+\langle g(\widetilde{\boldsymbol{b}}, \boldsymbol{P}(\widetilde{\boldsymbol{\tau}})), \mathbf{1}\rangle .
$$

To be more specific, let us introduce the quantities involved in writing the explicit functional form of $\mathbb{D}$, $\boldsymbol{f}$, and $\boldsymbol{g}$. We define:

$$
\begin{aligned}
& m_{1, i s}=\frac{1-x_{i, s-1}}{2 a} w_{i s}+\frac{1+x_{i, s-1}}{2}, \quad m_{2, i s}=\frac{1-x_{i, s-1}}{2 a} y_{i s} A_{s} \\
& c_{1, i s}=L^{b} m_{1, i s}+L^{h} h_{i} m_{2, i s}, \quad c_{2, i s}=L^{b} m_{2, i s} .
\end{aligned}
$$

A direct calculation from the definition of $\mathscr{F}$ gives

$$
\begin{aligned}
& \mathbb{D}_{i s, i s}=\left\{\begin{array}{ll}
-\rho^{s} c_{2, i s}, & K_{i s} \in[-a, a], \\
0, & K_{i s} \notin[-a, a],
\end{array} \quad g_{i s}= \begin{cases}-\rho^{s} L^{h} h_{i} m_{1, i s}, & K_{i s} \in[-a, a], \\
0, & K_{i s}<-a, \\
-\rho^{s} L^{h} h_{i}, & K_{i s}>a,\end{cases} \right. \\
& f_{i s}=\frac{1}{2} \begin{cases}\rho^{s}\left(c_{1, i s}-\frac{1}{|\alpha|}\right), & K_{i s} \in[-a, a], \\
\frac{\rho^{s}}{|\alpha|}, & K_{i s}<-a, \\
\rho^{s}\left(L^{b}-\frac{1}{|\alpha|}\right), & K_{i s}>a,\end{cases}
\end{aligned}
$$

\subsection{Optimal policy}

To compute optimal tax values we propose the following algorithms to find the optimal boat ramp fees, $\tau_{i s}^{*}$ for each lake $i$ in season $s$, that map from the optimal number of trips to each lake, $b_{*, i s}$.

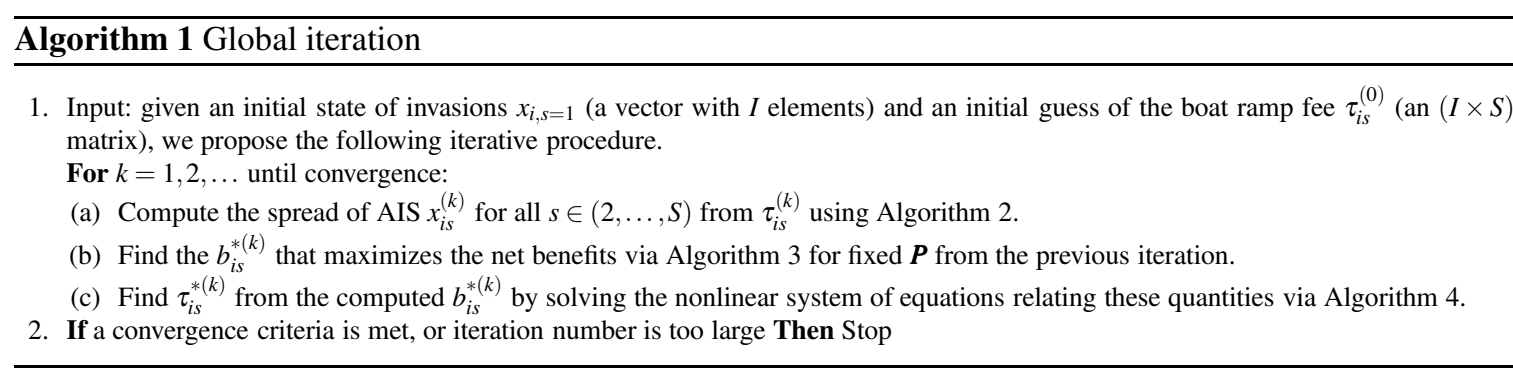

We now rewrite $L_{i s}$ as a linear function of $b_{i s}$ plus some "noise". To do this, let us introduce $A_{i s}$ - the average of $Q_{i s n t}$ with respect to $n$ and $t$ or the share of boater trips where the boater removes an invasive species from an invaded lake, namely.

$$
A_{i s}=\frac{1}{N T} \sum_{n} \sum_{t} Q_{i s n t}=\frac{1}{N T} \sum_{n} \sum_{t}\left[\sum_{j \neq i} \mu x_{j(s-1)} P_{j s n(t-1)}\right] .
$$

Then for $L_{i s}$ we have,

$$
L_{i s}=A_{i s} b_{i s}+\underbrace{\sum_{t} \sum_{n}\left(Q_{i s n t}-A_{i s}\right) P_{i s n t}}_{g_{i s}}
$$


In the algorithms that follow, the second term in the right side above is lagged, i.e. taken from the previous iteration. As a result, e can write $K_{i s}$ as a linear function of $\boldsymbol{b}$ :

$$
K_{i s}=\gamma A_{i s} b_{i s}+\gamma g_{i s}(\boldsymbol{b}, \boldsymbol{P})+\lambda_{i s} .
$$

where $A_{i s} b_{i s}$ is the average propagule pressure on lake $i$, i.e. the average number of trips to lake $i$ that carry an invasive species in season $s$, and $g_{i s}$ is a measure of the deviance across trip occasions from the average propagule pressure.

The algorithms described below, follow the steps outlined in the Global Iteration Algorithm 1
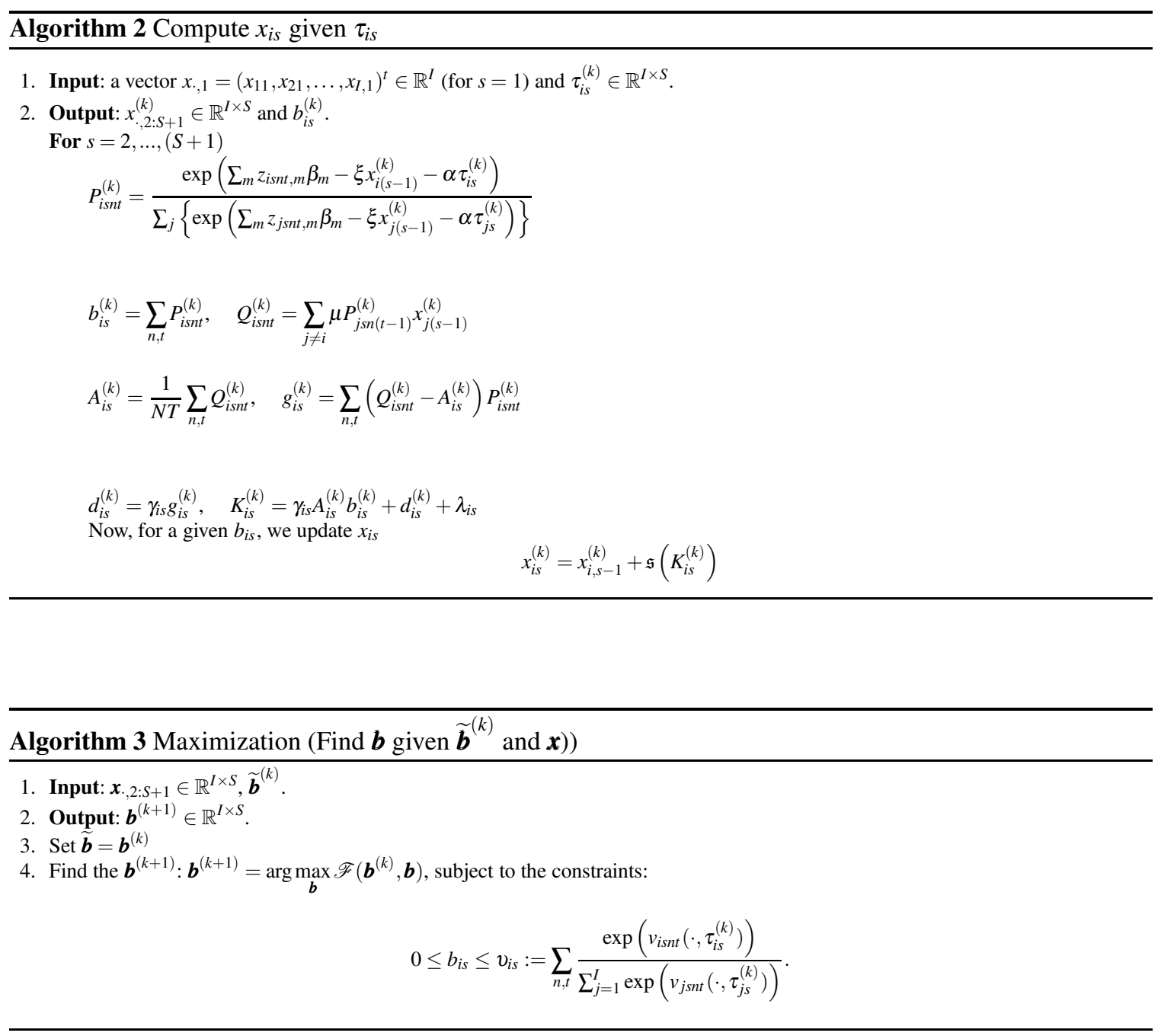

Notice that $\mathscr{F}(\widetilde{\boldsymbol{b}}, \cdot)$ is a quadratic functional for fixed $\widetilde{\boldsymbol{b}}$ with negative definite diagonal matrix $\mathbb{D}$. The solution then to the optimization problem in Algorithm 3, is given by (see Deutsch [20, Theorem 4.1, p.43])

$$
b_{i s}^{(k+1)}= \begin{cases}0, & {\left[\mathbb{D}^{-1} \boldsymbol{f}\right]_{i s}<0} \\ {\left[\mathbb{D}^{-1} \boldsymbol{f}\right]_{i s},} & 0 \leq\left[\mathbb{D}^{-1} \boldsymbol{f}\right]_{i s} \leq v_{i s} \\ u_{i s}, & {\left[\mathbb{D}^{-1} \boldsymbol{f}\right]_{i s}>v_{i s} .}\end{cases}
$$




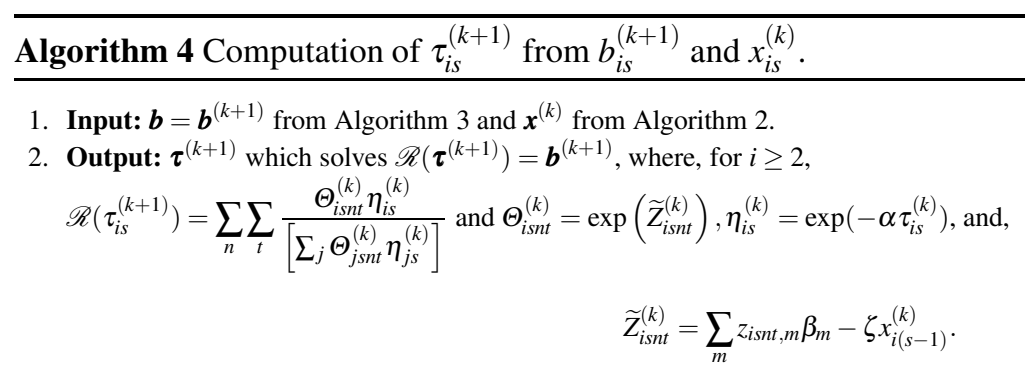

This nonlinear system is solved by the following iterative method:

Set $\eta_{1 s}=1\left(\right.$ or $\left.\tau_{1 s}^{(k)}=0\right), S=2, \ldots, S+1$. This reduces the number of unknowns, but number of equations is also reduced as $b_{1 s}=N T-\sum_{i \geq 2} b_{i s}$.

Set an initial guess $\tau_{i s}^{(0)}$ (which gives us $\eta_{i s}^{(0)}$ ).

For $q=1,2, \ldots$ until convergence:

For $s=2, \ldots, S+1, i=2, \ldots, I$ solve for $\eta_{i s}^{(q)}$

$$
\mathfrak{f}_{i s}\left(\eta^{(q)}\right):=\boldsymbol{b}_{i s}-\sum_{n} \sum_{t} \frac{\Theta_{i s n t} \eta_{i s}^{(q)}}{\Theta_{i s n t} \eta_{i s}^{(q)}+\sum_{j \neq i} \Theta_{j s n t} \eta_{j s}^{(q-1)}}=0
$$

If a convergence criteria is met, Then Stop

A comment on the solution of the nonlinear equations given in Algorithm 4 (see (12) $)$ is in order. By a closer inspection of the functions $\mathfrak{f}_{i s}(\cdot)$ on the right side of this equation we observe that $\mathfrak{f}_{i s}^{\prime}<0$. Furthermore, the upper bounds on $\boldsymbol{b}$ given in (9) guarantee that $\mathfrak{f}_{i s}(0)>0$ and $\mathfrak{f}_{i s}(1)<0$ whenever $\eta_{i s}^{(q-1)} \in(0,1)$. Hence, $\eta_{i s}^{q} \in(0,1)$ is unique (from the monotonicity of $\left.\mathfrak{f}_{i s}(\cdot)\right)$ and this guarantees that $\tau_{i s}^{q}$ is also unique and positive.

\section{Discussion and conclusions}

These algorithms allow us to empirically estimate the optimal spatial-dynamic policies to maximize the value of lakes net of damages from invasive species. To interpret these results we focus on the cases where $i$ and $s$ are such that $K_{i s} \in[-a, a]$. In these cases the optimal number of boating trips are defined as

$$
b_{i s}^{*}=\frac{\frac{1}{|\alpha|}-\mathscr{L}^{b}\left(\frac{\left(1-x_{i(s-1)}\right)}{2 a} w_{i s}+\frac{1+x_{i(s-1)}}{2}\right)-\mathscr{L}^{h} h_{i}\left(\frac{1-x_{i(s-1)}}{2 a}\right) y_{i s} A_{i s}}{\mathscr{L}^{b}\left(\frac{1-x_{i(s-1)}}{2 a}\right) y_{i s} A_{i s}} .
$$

We can simplify equation (13) so that the optimal number of trips to each lake occurs when

$$
\mathscr{L}^{b}\left(x_{i s}-x_{i(s-1)}\right)+\mathscr{L}^{h} h_{i} \frac{\partial x_{i s}}{\partial b_{i s}}=\frac{1}{|\alpha|} .
$$

The left-hand side of equation (14) is the expected marginal costs from boating. $\mathscr{L}^{b}\left(x_{i s}-x_{i(s-1)}\right)$ is the expected marginal damages to boaters from the spread of AIS and $\mathscr{L}^{h} h_{i} \frac{\partial x_{i s}}{\partial b_{i s}}$ is the marginal damages to homeowners from boaters spreading AIS. The right-hand side of equation (14) is the marginal benefit of boating.

Notice when the propagule pressure $A_{i s} \rightarrow 0$ optimal $b_{i s}^{*} \rightarrow \infty$. Since we do not allow the optimal boat ramp fee to be negative $b_{i s}^{*}=v_{i s}$ and $\tau_{i s}^{*}=0$. This makes intuitive sense; if AIS dispersal from boaters is small, then the optimal policy is to have no boat ramp fee. On the other hand when the benefits from boating are 
lower than the damages from boating then the optimal number of boating trips might reach the lower bound, which is zerd 3 . The lower bound of boating trips corresponds to an optimal boat ramp fee that approaches infinity, $\tau_{i s}^{*} \rightarrow \infty$. This also makes intuitive sense; if the damages from AIS are worse than the benefits from boating it makes sense to close the lake to boating.

\subsection{A numerical example}

Next, we provide a simple numerical illustration of how the proposed method works. We consider a system with 2 lakes, 10 boaters, 2 seasons, and with 11 days per season, i.e. $(I, S, N, T)=(3,1,10,11)$. For the rest of the model parameters, we have set $\mathscr{L}^{b}=100, \mathscr{L}^{h}=1400, \gamma=5, \xi=0.1, f=0.03, h_{i}=1$, for $i=1, \ldots, I$, $\lambda_{i s}=-0.001, r=0.5 \times 10^{-1}, x_{\cdot s=1}=0.5, \tau_{i, s}=1.0$, and $\tilde{Z}_{i s n t} \sim$ Uniform $(-2,-1)$. In Table 1 we show the optimal number of boating trips, tax values, invaded probabilities and the total total benefits value. The data are for three simulations with varying $\alpha$ in which we have the optimal value of the boating trips $b_{i s}$ to be at the upper bound, at the lower bound, or in the middle.

Table 1 Optimal number of boating trips for varying values of the parameter $\alpha$

\begin{tabular}{|c|c|c|c|}
\hline$\alpha$ & 0.010 & 0.133 & 0.015 \\
\hline upper bound (U) on $b_{i \in\{2,3\}, s=2}$ & $36.16,36.39$ & $53.53,53.81$ & $53.27,53.56$ \\
\hline lower bound (L) on $b_{i \in\{2,3\}, s=2}$ & 0,0 & 0,0 & 0,0 \\
\hline optimal boating $b_{i \in\{2,3\}, s=2}^{*}$ & $36.16,36.39$ & $34.74,33.02$ & $0.00,0.00$ \\
\hline optimal tax $\tau_{i \in\{2,3\}, s=2}^{*}$ & $0.02,0.02$ & $57.64,63.72$ & $375.04,374.71$ \\
\hline invaded prob. $x_{i \in\{2,3\}, s=2}^{*}$ & $0.83,0.83$ & $0.74,0.74$ & $0.75,0.75$ \\
\hline $\mathscr{F}\left(b_{i s}=L\right), \mathscr{F}\left(b_{i s}=U\right)$ & $-8802,-1522$ & $-8054,-3678$ & $-1115,-3479$ \\
\hline $\mathscr{F}\left(b_{i s}=b_{i s}^{*}\right)$ & -1522 & -1669 & -1115 \\
\hline
\end{tabular}

\section{Conclusions}

In conclusion, we have developed a novel method to solve for the optimal management of a complex spatialdynamic process (the spread of AIS) with an endogenous diffusion mechanism. The benefits of our method include an analytical solution for the optimal number of trips to each lake in each season and an iterative method to solve for the spatial-dynamic optimal boat ramp fee $\boldsymbol{\tau}$ from $\boldsymbol{b}$ that is likely to converge. A careful look at the algorithms given here show that the main computational cost is in evaluating the function $\mathscr{F}$. Applying this novel framework to empirical data, to other migration processes, as well as the mathematical analysis of the proposed algorithms are subject of current and future research.

\section{References}

1. D.S. Wilcove, D. Rothstein, J. Dubow, A. Phillips, E. Losos, BioScience 48(8), 607 (1998). DOI 10.2307/1313420

2. O.E. Sala, F.S. Chapin, J. Armesto, E.L. Berlow, J. Bloomfield, R. Dirzo, E. Huber-Sanwald, L.F. Huenneke, R.B. Jackson, a. Kinzig, R. Leemans, D.M. Lodge, H. Mooney, M. Oesterheld, N.L. Poff, M.T. Sykes, B.H. Walker, M. Walker, D.H. Wall, Science 287(5459), 1770 (2000). DOI 10.1126/science.287.5459.1770. URL http://www.ncbi.nlm.nih.gov/pubmed/10710299

3. T.P. Moorhouse, D.W. Macdonald, WIREs Water 2(1), 1 (2015). DOI 10.1002/wat2.1059

4. J.D. Rothlisberger, W.L. Chadderton, J. McNulty, D.M. Lodge, Fisheries 35(3), 121 (2010). DOI 10.1577/1548-8446-35.3.121. URL http://www .tandfonline.com/doi/abs/10.1577/1548-8446-35.3.121

5. R.S. Epanchin-Niell, M.B. Hufford, C.E. Aslan, J.P. Sexton, J.D. Port, T.M. Waring, Frontiers in Ecology and the Environment 8(4), 210 (2010). DOI 10.1890/090029. URL http://doi.wiley.com/10.1890/090029

6. K.Y. Zipp, D.J. Lewis, B. Provencher, M.J.V. Zanden, Land Economics 95(1) (2019)

3 Clearly, allowing a negative number of boating trips would not be realistic. 
7. E.J. Horsch, D.J. Lewis, Land Economics 85(3), 391 (2009).

DOI $\quad 10.1353 /$ lde.2009.0042.

URL http://le.uwpress.org/content/85/3/391.abstract

8. B. Provencher, D.J. Lewis, K. Anderson, Journal of Environmental Economics and Management 64(2), 169 (2012). DOI 10.1016/ j.jeem.2012.04.002. URL http://linkinghub.elsevier.com/retrieve/pii/S009506961200037X

9. D.J. Lewis, B. Provencher, B. Beardmore, Ecological Economics 114, 141 (2015). DOI 10.1016/j.ecolecon.2015.03.025. URL http://linkinghub.elsevier.com/retrieve/pii/S0921800915001421

10. W. Brock, A. Xepapadeas, Journal of Economic Dynamics and Control 32(9), 2745 (2008). DOI 10.1016/j.jedc.2007.08.005

11. W. Brock, A. Xepapadeas, Journal of Environmental Economics and Management 59(2), 149 (2010). DOI 10.1016/j.jeem.2009.07. 003. URL http://dx.doi.org/10.1016/j.jeem.2009.07.003

12. J.N. Sanchirico, H.J. Albers, C. Fischer, C. Coleman, Environmental and Resource Economics 45(4), 517 (2010). DOI 10.1007/ s10640-009-9326-0

13. M.E. Eiswerth, G.C. van Kooten, The American Journal of Agricultural Economics 84(5), 1317 (2002)

14. J. Hof, Natural Resource Modeling 11(3), 197 (1998).
http://doi.wiley.com/10.1111/j.1939-7445.1998.tb00308.x

DOI $10.1111 /$ j.1939-7445.1998.tb00308.x. URL

5. R. Horan, C.A. Wolf, E.P. Fenichel, K.H. Mathews, Review of Agricultural Economics 27(3), 483 (2005). DOI 10.1111/j. 1467-9353.2005.00248.x

16. B. Leung, D.M. Lodge, D. Finnoff, J.F. Shogren, M.A. Lewis, G. Lamberti, Proceedings of the Royal Society B: Biological Sciences 269(1508), 2407 (2002). DOI 10.1098/rspb.2002.2179

17. K.E. Train, Cambridge University Press pp. 1-388 (2009). DOI 10.1017/CBO9780511753930. URL http://ebooks.cambridge.org/ref/id/CB09780511753930

18. C. Chivers, B. Leung, Journal of Applied Ecology 49(5), 1113 (2012). DOI 10.1111/j.1365-2664.2012.02183.x

19. L. Bruckerhoff, J. Havel, S. Knight, Hydrobiologia 746(1), 113 (2014). DOI 10.1007/s10750-014-1947-9. URL http://link.springer.com/10.1007/s10750-014-1947-9

20. F. Deutsch, Best approximation in inner product spaces, CMS Books in Mathematics/Ouvrages de Mathématiques de la SMC, vol. 7 (Springer-Verlag, New York, 2001). DOI 10.1007/978-1-4684-9298-9. URL https://doi.org/10.1007/978-1-4684-9298-9 